\title{
Poly(ethyleneglycol) in electrospray ionization (ESI) mass spectrometry
}

\author{
S. Varray, J.-L. Aubagnac, F. Lamaty, R. Lazaro, J. Martinez and C. Enjalbal*
}

Laboratoire des Aminoacides, Peptides et Protéines, UMR 5810, Université Montpellier II, 34095 Montpellier Cedex 05, France

* Corresponding author: enjalbal@crit.univ-montp2.fr

Poly(ethyleneglycol) (PEG) substituted by various organic moities were analyzed by electrospray ionization (ESI) mass spectrometry in order to determine the best experimental protocol allowing reproducible, sensitive and informative spectra in the search of completely automated high throughput identification of libraries generated by the liquid-phase methodology. The nature of the eluent and of the PEG derivatization as well as the value of the sampling cone voltage were investigated by comparing the recorded oligomer distributions.

\section{ABBREVIATION}

ESI: Electrospray ionization.

FIA: Flow injection analysis.

LC/MS: Liquid chromatography / mass spectrometry. MALDI: Matrix assisted laser desorption ionization. NMR: Nuclear magnetic resonance.

PEG: Poly(ethyleneglycol).

\section{Introduction}

Taking advantages of both homogeneous and solid-phase chemistries, soluble polymers such as poly(ethyleneglycol) (PEG) have been investigated as an attractive alternative to 
standard resins to perform supported organic reactions [1]. In contrast to the most popular solid-phase methodology, solubilisation of the substituted polymer in spectroscopic solvents allows the characterization by NMR and mass spectrometry of the growing structures without their prior release in solution. Matrix assisted laser desorption ionization (MALDI) [2] and electrospray ionization (ESI) [3] mass spectrometry were investigated for direct supported reaction monitoring on PEG [4-7]. The control of reaction completion by mass spectrometry only required the comparison of the theoretical and observed mass increments between the starting material and the product corresponding to the mass of the anchored structure.

Each of the chosen techniques, MALDI and ESI, presents advantages and drawbacks. Indeed, at each step of the synthetic scheme, the shift of the PEG distribution was easily followed by MALDI which provided positive ion mass spectra showing solely singly charged molecular ions [4]. Nevertheless, a chemical reactivity imparted by the common use of acidic matrices was reported in the case of PEG functionalized by acid-sensitive materials leading to erroneous data interpretation [5]. The choice of the matrix was thus governed by the nature of the studied samples. Atmospheric pressure ionization methods including ESI do not exhibit such limitations associated to the presence of a matrix. However, the recorded ESI mass spectra were more complex than those obtained by MALDI due to the overlapping of the oligomer distribution with multiply charged cluster ions $[6,7]$.

The synthesis of combinatorial parallel libraries on soluble polymers was recently described in the literature $[8,9]$ and prompted us to investigate the high throughput profiling of collections of substituted PEG by mass spectrometry. Despite the straightforward MALDI mass spectra interpretation obtained in the context of the step by step monitoring of supported organic reactions, ESI was preferred for several reasons. First, no optimization of the mass spectrometric experiment was required to adapt the conditions to the studied samples as discussed above with MALDI. Thus, the same system could be applied to analyze a wide range of structurally different compounds with reduced sample handling and acquisition time. Second, atmospheric pressure ionization methods constitute up to now the sole techniques allowing convenient highly informative LC/MS experiments [10]. Finally, fully automated open access systems performing both high throughput flow injection and LC/MS ESI analyses were recently reported in the literature $[11,12]$.

In order to validate such methodology to PEG based samples, specific problems due to the presence of mixtures of oligomers must be addressed. Experimental parameters including the nature of the eluent, the value of the sampling cone voltage as well as the effect of the polymer substituent were investigated and are discussed in the scope of obtaining sensitive, reproducible and informative mass spectra.

\section{Experimental}

Syntheses of the compounds are described elsewhere [13].

ESI mass spectra were recorded on a Platform II quadrupole mass spectrometer (Micromass, Manchester, UK) fitted with an electrospray ion source. The mass spectrometer was calibrated in the positive ion mode using a mixture of $\mathrm{NaI}$ and CsI. Data were acquired in the scan mode from $\mathrm{m} / \mathrm{z}, 200$ to 2500 in $5 \mathrm{~s}$. Ten scans were summed to produce the final spectra. Samples were dissolved in different eluent such as $\mathrm{CH}_{3} \mathrm{CN}, \mathrm{CH}_{3} \mathrm{CN} / \mathrm{H}_{2} \mathrm{O}(50: 50, \mathrm{v} / \mathrm{v}), \mathrm{CH}_{3} \mathrm{CN} / \mathrm{H}_{2} \mathrm{O} / \mathrm{HCO}_{2} \mathrm{H}$ (49:49:2, v/v), $\mathrm{CH}_{3} \mathrm{OH}, \mathrm{CH}_{3} \mathrm{OH} / \mathrm{H}_{2} \mathrm{O}$ (50:50, v/v), $\mathrm{CH}_{3} \mathrm{OH} /$ $\mathrm{H}_{2} \mathrm{O} / \mathrm{HCO}_{2} \mathrm{H}(49: 49: 2, \mathrm{v} / \mathrm{v})$, and infused into the ESI source at a flow rate of $60 \mu \mathrm{l} / \mathrm{min}$. Voltages were set at $+3.5 \mathrm{kV}$ for the capillary and adjusted for the sampling cone. The source was heated at $120^{\circ} \mathrm{C}$.

\section{Results and discussion}

Although identification of the various oligomers from PEG mixtures was not required to monitor step by step liquidphase reactions by mass spectrometry, this problem must be tackled to reveal which experimental parameters influence the ESI spectra. The structures of the most commonly used soluble polymers, $\mathrm{PEG}_{2000}$ and $\mathrm{PEG}_{3400}$ with an average mass distribution centered around 2000 and 3400 respectively, are given in scheme 1. Each oligomer possessing a define number $n$ of ethyleneglycol unit $\left(-\mathrm{CH}_{2}-\mathrm{CH}_{2}-\mathrm{O}-\right)$ exhibits an hydroxyl functionality at both ends which were substituted simultaneously by an organic moiety R.

As detailed in our previous works [6,7], the mass increment between native and derivatized PEG noted $\triangle \mathrm{M}$ corresponds to twice the mass $\mathrm{M}$ of the substituent $\mathrm{R}$ minus two hydrogens divided by the charge state $(z)$ of the multiply charged ion distribution. For instance, the mass difference $\Delta \mathrm{M}$ between singly, doubly and triply protonated ions clusters of PEG and derivatized PEG should be 2(M-1), $2(\mathrm{M}-1) / 2$ and $2(\mathrm{M}-1) / 3$, respectively. The attribution of the charge state was deduced from the mass difference between two consecutive ions belonging to the same cluster. Indeed, these two ions must be separated by 44 Th [14] (ethyleneglycol unit) in the singly charge distribution, by $44 / 2$ Th on the doubly charged species and more generally by 44/z Th on any clusters exhibiting $z$ charges. The degree of polymerization $n$ was then calculated for the various studied compounds listed in table I according to the formula shown in scheme 1 .

The most general case was considered with ionization occurring by both protonation and cationization with sodium. On any PEG distribution possessing a charge state $z$, all ions of that cluster can be described as cationized by y sodium cations and $(z-y)$ protons. For any aforementioned ions, the masses of the two terminal moieties OR and R correspond to $(2 \mathrm{M}+16) / z$ Th. To deduce the mass of 

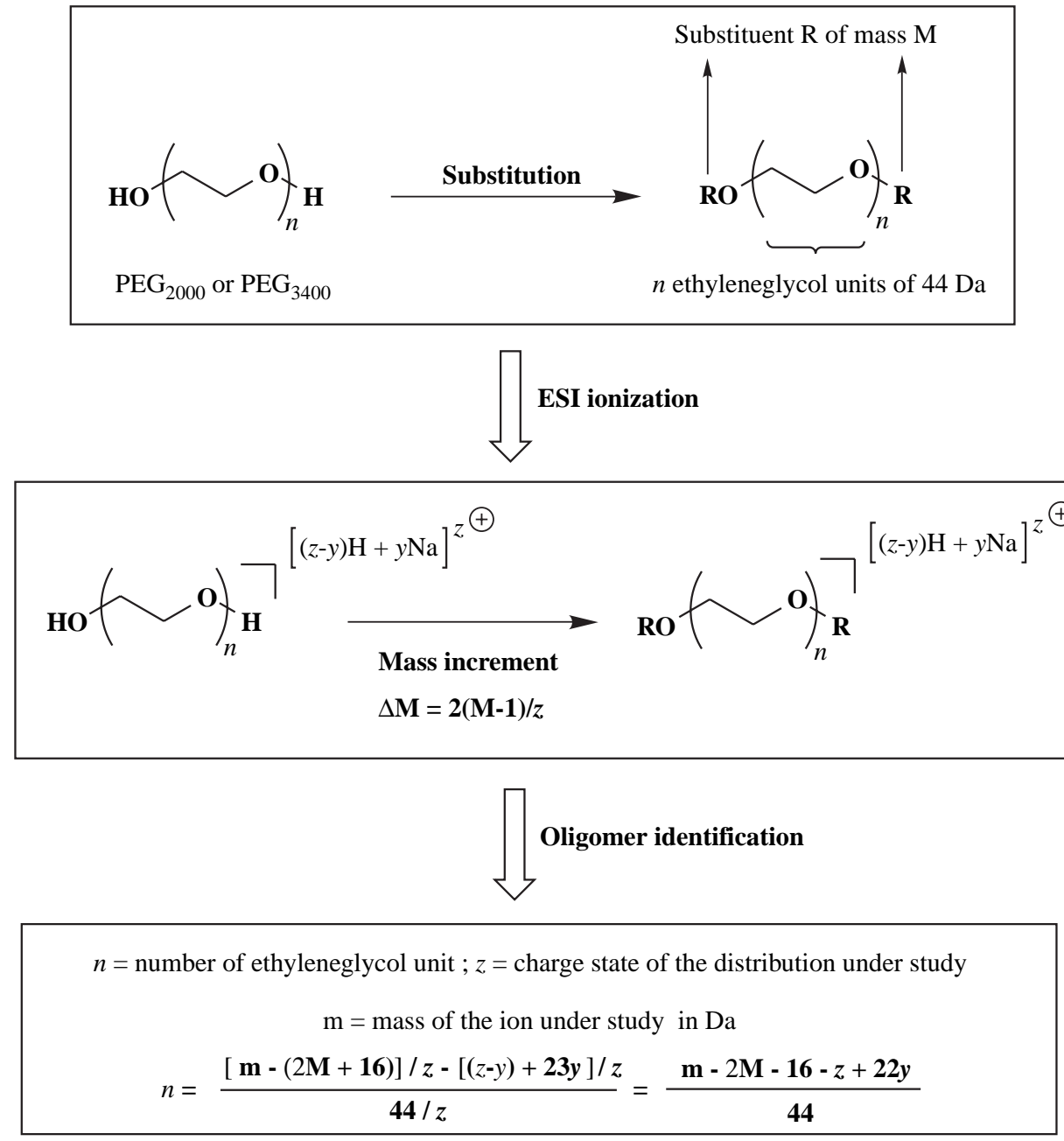

Scheme 1

ethyleneglycol skeleton in a chosen oligomer, one should subtract to the recorded ion at a charge state $z$ the masses of the two terminal moieties $((2 \mathrm{M}+16) / z \mathrm{Th})$ as well as the masses of the protons and sodium cations ([(z$y)+23 y] / z$ Th). The number of ethyleneglycol unit can then be obtained by dividing by the mass of the monomer i.e. $44 / z$ at the charge state under study. The formula is given in scheme 1 .

Positive ion mass spectra were systematically acquired except when anions were anchored onto the PEG backbone. In that case, negative ion mass spectra were successfully recorded (compound $\mathbf{6}$, table I).

The effects of eluent, sampling cone voltage and PEG substituent on ionization of the compounds (table I) were thereafter investigated by comparing the detected oligomers, their charge states and the possible presence of sodium adducts. To illustrate these studies, only compounds $\mathbf{1}$ to $\mathbf{4}$ will be discussed.
Table I. Studied samples.

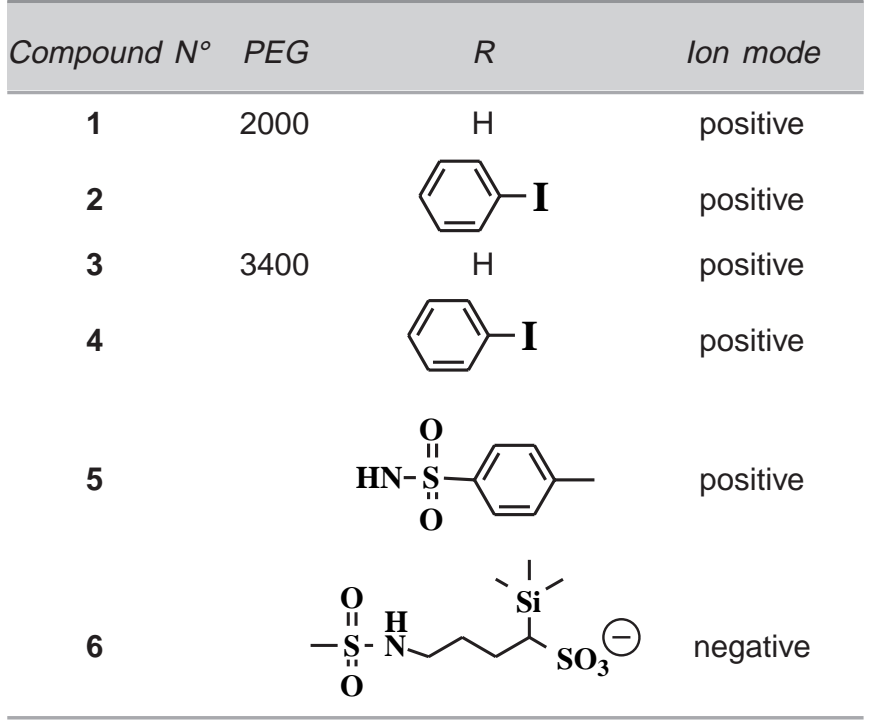




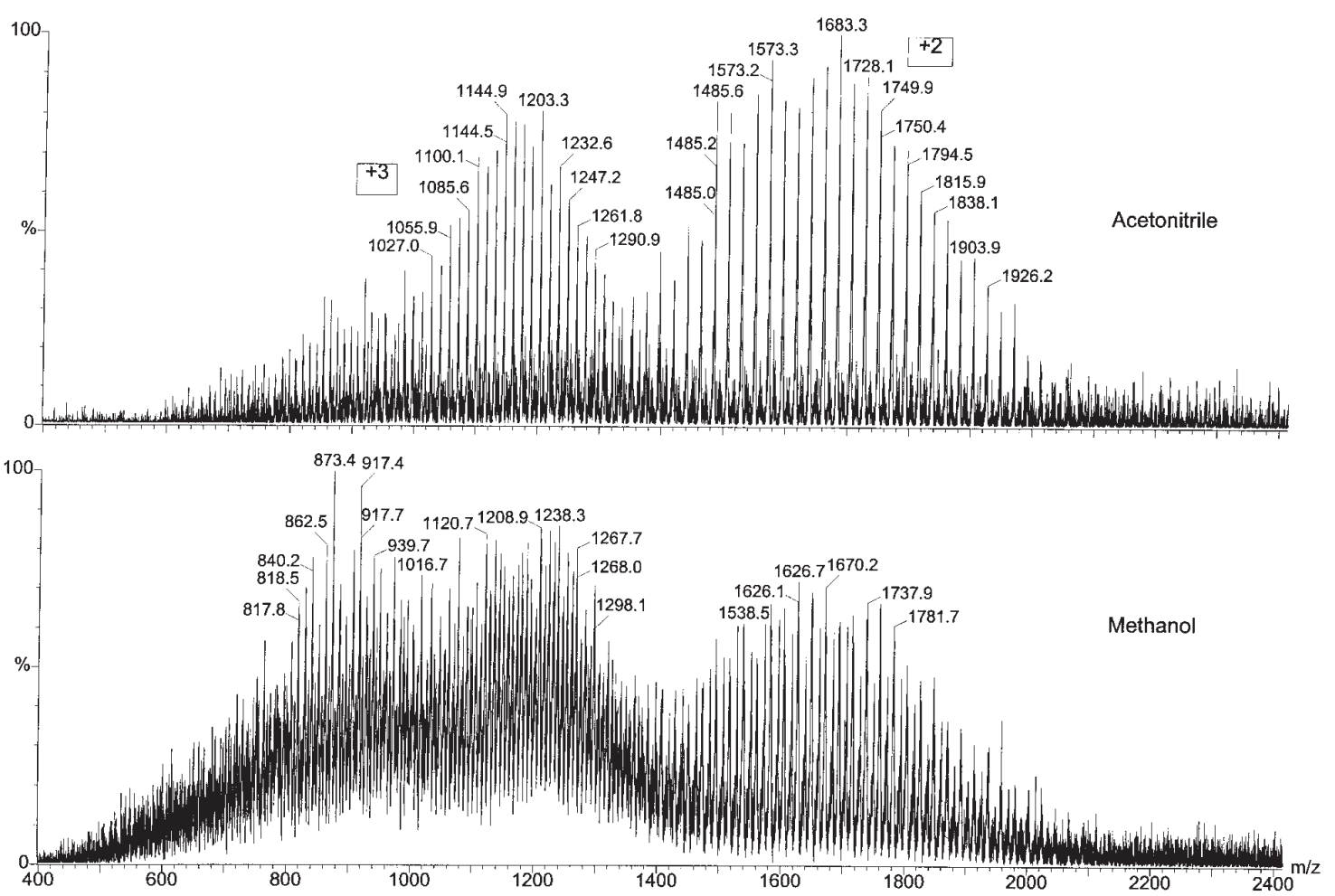

Figure 1. Positive ion ESI mass spectra of $\mathrm{PEG}_{3400}$ at $30 \mathrm{~V}$.

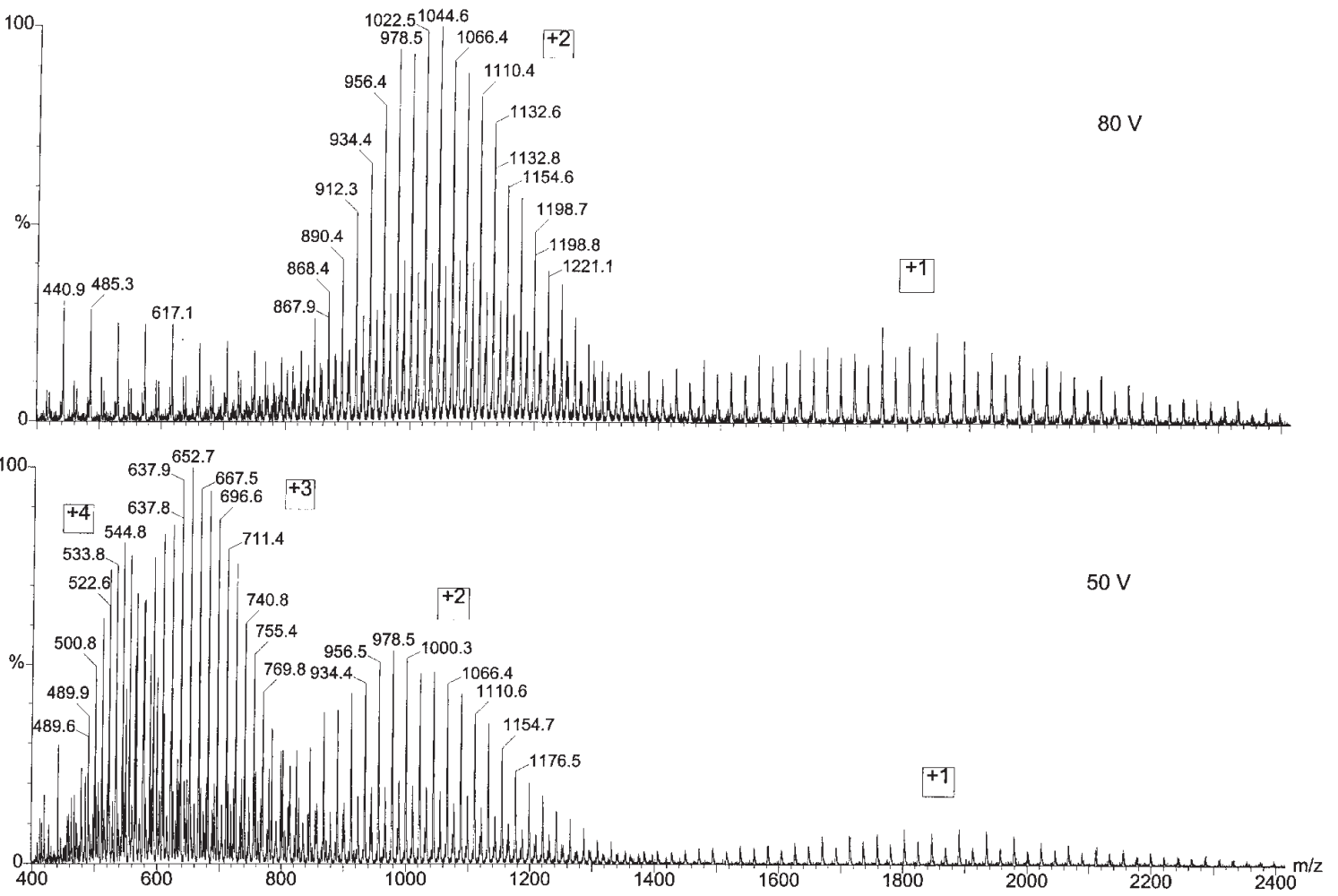

Figure 2. Positive ion ESI mass spectra of $\mathrm{PEG}_{2000}$ dissolved in $\mathrm{CH}_{3} \mathrm{CN} / \mathrm{H}_{2} \mathrm{O} / \mathrm{HCO}_{2} \mathrm{H}$. 
Table II. Oligomer identification in ESI mass spectra of various PEG samples.

\begin{tabular}{ccccccc}
\hline & & \multicolumn{5}{c}{ Charge state } \\
$N^{0}$ & Eluent & $\mathrm{CV}$ & +1 & +2 & +3 & +4 \\
\hline 1 & $\mathrm{CH}_{3} \mathrm{CN}$ & no & $+\mathrm{Na}^{\dagger}$ & $+\mathrm{H}$ & & \\
& & effect & $(33-52)^{\ddagger}$ & $(38-60)$ & - & - \\
& & & & \\
$\mathrm{CH}_{3} \mathrm{CN} / \mathrm{H}_{2} \mathrm{O} /$ acid & $50 \mathrm{~V}$ & & $+\mathrm{H}$ & $+\mathrm{H}$ & $+\mathrm{H}$ \\
& & & - & $(38-60)$ & $(40-54)$ & $(40-54)$
\end{tabular}

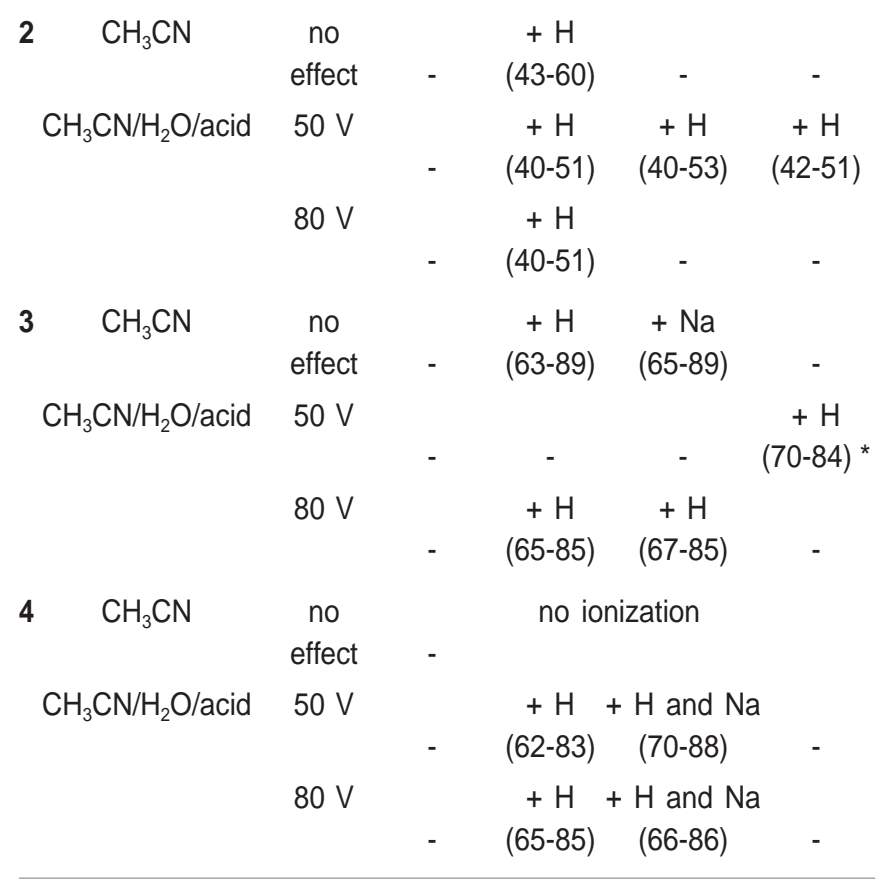

$\dagger$ ionization by protonation or cationization

$¥$ number of ethyleneglycol units

* charge states +5 and +6 also detected

First of all, it should be emphasized that all studied experimental parameters were not acting independently upon ESI ionization. The combined effects of the nature of the eluent and the value of the sampling cone voltage on compounds $\mathbf{1}$ to $\mathbf{4}$ are summarized in table II.

For all samples, acetonitrile provided far better ionization than the corresponding methanolic solution. As an illustration, the positive ion spectra of compound $\mathbf{3}\left(\mathrm{PEG}_{3400}\right)$ recorded in acetonitrile and methanol are shown in figure 1. Besides, variation of the sampling cone voltage affected significantly the spectra except when pure acetonitrile was used (Tab. II). Although this eluent looked attractive, poor ionization efficiencies were observed for derivatized PEG, especially in the case of $\mathrm{PEG}_{3400}$ (see for instance compound $\mathbf{4}$ in table II). Under such conditions, the standard ESI eluent,
$\mathrm{CH}_{3} \mathrm{CN} / \mathrm{H}_{2} \mathrm{O} / \mathrm{HCO}_{2} \mathrm{H}(49: 49: 2$, v/v), was chosen because efficient ionization was recovered for all studied PEG samples. In the case of PEG functionalized by acid-sensitive materials, the use of the aforementioned eluent system provided extensive product degradation as observed in MALDI experiments carried out with acidic matrices. These samples were not suitable for automated FIA analyses and were thus characterized by direct infusion of non acidic solutions into the ESI source.

Nevertheless, this choice brought some drawbacks. First, the presence of formic acid favored high charge states and the possibility of overlapping distributions hampering ion assignment was increased. Second, the value of the sampling cone voltage was found to affect greatly the profile of the ESI spectra. For all studied compound dissolved in acidic acetonitrile solutions high charge state distributions were detected at sampling cone voltages inferior to $50 \mathrm{~V}$ whereas low protonation or cationization was observed for sampling cone voltages superior to $80 \mathrm{~V}$ (Tab. II). Instead of a steady shift in the charge state distribution as commonly encountered in ESI [15], a sudden change was observed around $60-70$ V. Such behavior is illustrated in figure 2 which displays the positive ion ESI spectra of compound $\mathbf{1}$ recorded at $50 \mathrm{~V}$ and $80 \mathrm{~V}$. Since limited protonation or cationization was occurring at high sampling cone voltage values providing rather simple spectra, $80 \mathrm{~V}$ was chosen for routine analyses. It should be noted that with this sampling cone voltage value the observed ions ranged from 800 to $2500 \mathrm{Th}$. Mass spectrometers fitted with an analyzer limited to $1600 \mathrm{Th}$, generally devoted to combinatorial chemistry, would provide truncated spectra displaying high charge state distributions only. Under such conditions, the presence of a unique ion cluster within the recorded mass range could be envisaged and compound identification would be based on a single mass increment measurement generating the risk of erroneous data interpretation.

In a spectrum, the oligomer distribution was slightly narrower for higher charge states. For compound 1, PEG chains possessing 38 to 60 ethyleneglycol units were detected as doubly charged species whereas structures containing only 40 to 54 monomers were observed in the tri- and tetra-protonated ion clusters (Tab. II). Moreover, similar ion distributions were recorded for every compound whatever the eluent or sampling cone voltage meaning that no discrepancy was observed for the ionization of such structurally related mixture components. As an example, the same oligomers possessing 38 to 60 ethyleneglycol units were evidenced on the doubly charged distribution of compound $\mathbf{1}$ for all performed experiments.

Finally, ionization was found to occur mainly by protonation. When cationization was detected, a single ion cluster in all charge state distributions was affected. No general trends were however evidenced from the studied samples, meaning that caution must be observed for data interpretations by identifying first few oligomers prior to mass increment calculations. 


\section{Conclusion}

We have shown in this preliminary study that PEG samples could be routinely analyzed by ESI mass spectrometry without requiring extensive experimental optimization. High sampling cone voltages and acidic acetonitrile eluents were chosen as optimum ionization conditions. Nevertheless, further research are under investigation to widen the scope to liquid-phase library characterization requiring a fully automated system.

\section{References}

1. Gravert, D. J.; Janda, K. D. Chem. Rev. 1997, 489.

2. Karas, M.; Glückmann, M. Schäfer, J. J. Mass Spectrom. 2000, $35,1$.

3. Gaskell, S. J. J. Mass Spectrom. 1997, 32, 677.

4. Thurmer, R.; Meisenbach, M.; Echner, H.; Weiler, A.; AlQawasmeh, R.; Voelter, W.; Korff U.; Schmitt-Sody, W. Rapid Communic. Mass Spectrom. 1998, 12, 398
5. Enjalbal, C.; Sauvagnat, B.; Lamaty, F.; Lazaro, R.; Martinez, J.; Mouchet, P.; Roux F.; Aubagnac, J-L. Rapid Communic. Mass Spectrom. 1999, 13, 1775.

6. Sauvagnat, B.; Enjalbal, C.; Lamaty, F.; Lazaro, R.; Martinez J.; Aubagnac, J-L. Rapid Communic. Mass Spectrom. 1998, 12, 1034.

7. Nativel, F.; Enjalbal, C.; Lamaty, F.; Lazaro, R.; Martinez J.; Aubagnac, J-L. Eur. Mass Spectrom. 1998, 4, 233.

8. Han, H.; Wolfe, M. M.; Brenner S.; Janda, K. D. Proc. Natl. Acad. Sci. USA 1995, 92, 6419.

9. Yeh C-M.; Sun, C-M. Tetrahedron Lett. 1999, 40, 7247.

10. Lee M. S.; Kerns, E. H. Mass Spectrom. Rev. 1999, $18,187$.

11. Zeng L.; Kassel, D.B. Anal. Chem. 1998, 70, 4380.

12. Dulery, B.D.; Verne-Mismer, J.; Wolf, E.; Kugel C.; Van Hijfte L. J. Chromatogr. B 1999, 725, 39.

13. Varray, $\mathrm{S}$; $\mathrm{PhD}$ Thesis in progress.

14. Cooks R. G.; Rockwood, A. L. Rapid Commun. Mass Spectrom. 1991, 5, 93.

15. Siudzdak G. in Mass spectrometry for biomolecules; London: Academic Press, 1996. 